\title{
Combining GPS, GIS, and Accelerometry: Methodological Issues in the Assessment of Location and Intensity of Travel Behaviors
}

\author{
Melody Oliver, Hannah Badland, Suzanne Mavoa, Mitch J. Duncan, and Scott Duncan
}

\begin{abstract}
Background: Global positioning systems (GPS), geographic information systems (GIS), and accelerometers are powerful tools to explain activity within a built environment, yet little integration of these tools has taken place. This study aimed to assess the feasibility of combining GPS, GIS, and accelerometry to understand transport-related physical activity (TPA) in adults. Methods: Forty adults wore an accelerometer and portable GPS unit over 7 consecutive days and completed a demographics questionnaire and 7-day travel log. Accelerometer and GPS data were extracted for commutes to/from workplace and integrated into a GIS database. GIS maps were generated to visually explore physical activity intensity, GPS speeds and routes traveled. Results: GPS, accelerometer, and survey data were collected for 37 participants. Loss of GPS data was substantial due to a range of methodological issues, such as low battery life, signal drop out, and participant noncompliance. Nonetheless, greater travel distances and significantly higher speeds were observed for motorized trips when compared with TPA. Conclusions: Pragmatic issues of using GPS monitoring to understand TPA behaviors and methodological recommendations for future research were identified. Although methodologically challenging, the combination of GPS monitoring, accelerometry and GIS technologies holds promise for understanding TPA within the built environment.
\end{abstract}

Oliver, Badland, and S. Duncan are with the Centre for Physical Activity and Nutrition Research, Auckland University of Technology, Auckland, New Zealand. Mavoa is with Massey University, Auckland, New Zealand. M. Duncan is with the Centre for Social Science Research, Central Queensland University, Rockhampton, Australia.
Keywords: accelerometry, environment, physical activity assessment, measurement

An emerging body of research linking physical activity engagement to the built environment has shown that urban design components can facilitate or inhibit different domains of physical activity behavior, such as transportrelated physical activity (TPA) (eg, walking and cycling for travel purposes). ${ }^{1,2}$ TPA presents the opportunity to engage in physical activity by integrating regular, habitual activity into the daily routines of individuals, thereby overcoming the time constraints reported by many as a major barrier to physical activity. ${ }^{3}$ The accumulation of TPA has been shown to reduce the risk of hypertension, diabetes, cardiovascular disease, and overall mortality. $^{4-7}$ These relationships highlight the potential public health advantages that can be gained by promoting TPA participation.

Built environment infrastructure, such as wellconnected and maintained footpaths, cycle lanes, and street networks, appear to be important facilitators of TPA engagement, ${ }^{8-10}$ and are recognized as key correlates in combination with time and distance factors. The majority of previous research in this area, however, has been reliant on subjective self-report methodologies, ${ }^{1}$ and there is a need for the utilization of objective measures in this field to overcome the shortcomings of self-reported data. ${ }^{11}$ The integration of multiple objective measures such as accelerometers, Global Positioning System (GPS) units, and Geographic Information Systems (GIS), show promise for objectively assessing geographical settings and associated domain-specific physical activity patterns. ${ }^{12}$

GPS monitoring is gaining in popularity among physical activity researchers due to its utility when implemented alongside objective measures of physical activity (accelerometers) and the built environment (GIS). When combined, these objective tools can be used to ascertain physical activity levels and the spatial location of activity while identifying key variables within the built environment that affect travel modes or activity level. A compre- 
hensive understanding of the environmental associations of travel modes and TPA is needed to inform the creation of environments that promote engagement in sustainable travel behaviors, and to provide further evidence of how urban design may influence human behavior and related health outcomes. Few studies have combined GPS and accelerometry data to assess PA behavior, ${ }^{12,13}$ and none have explicitly targeted TPA behaviors. Accordingly, the current study firstly investigates the feasibility of integrating GPS, accelerometer and GIS data, and secondly considers the utility of this approach for determining differences in mode of transportation and concurrent commute routes traveled. It is envisaged that the identification of methodological issues related to these technologies will contribute to the development of this emergent field of research.

\section{Methods}

\section{Sample and Setting}

A convenience sample of adults residing in North Shore City, Auckland, New Zealand and working at Auckland University of Technology (North Shore Campus) were recruited to participate in the current study. All participants took part in a 7-day monitoring period between January and March 2008. Individuals aged less than 20 years, with severe walking mobility restrictions, or with minimal command of the English language were excluded from the study. Those living outside of the North Shore City region were also excluded as this was the area captured by the GIS database. All participants provided informed consent. Ethical approval to conduct the study was provided by the host institution ethics committee.

\section{Protocol and Measures}

After registering with the study, participants met with a trained research assistant to receive measurement instruments, travel logs, and instructions about instrument usage. Participants completed a travel and compliance log during the time they wore the accelerometer and GPS units. Over the 7-day measurement period, participants reported the time they woke up and went to bed, their travel mode to and from work, trip chaining activities before and after work, and time and activity engaged in when the accelerometer was not worn.

\section{GPS and Accelerometer Protocols}

Participants were provided with an Actical accelerometer (Mini-Mitter, Respironics Inc. Company, Bend, OR) that was attached to an elastic belt and set to record activity data in 15-second epochs. Individuals were asked to wear the belt at their waist with the monitors placed above the right iliac crest for the entire monitoring period and to remove the belt (containing both accelerometer and GPS units) only when sleeping or bathing. The Actical accelerometer has been shown to be both reliable ${ }^{14}$ and valid $^{15,16}$ for estimating physical activity in adults. In addi- tion, the 7-day monitoring protocol has been established as a reliable measure of habitual PA. ${ }^{17}$ A Trackstick II GPS unit (Telespial Systems, Marina Del Rey, CA) was also attached to the accelerometer belt. These small and portable (unit size $11.43 \times 3.18 \times 1.9 \mathrm{~cm}$ ) GPS units can continuously record data on time, date, speed, altitude, direction, and spatial location to within $2.5 \mathrm{~m}$; however, they have not been assessed for their utility or accuracy in monitoring physical activity.

The Trackstick units were fitted with rechargeable NiMh batteries (PowerEx 1000 mAh, Maha Energy Corp, City of Industry, CA), and set to full power (normal) mode, with all other options left at the manufacturers' default settings. Using NiMh batteries, the Trackstick has a battery life of 3 to 5 days in the power save (low power) mode, and 24 hours in the full power mode. Data quality can be reduced in the low power mode, with reduced recording intervals and satellite acquisition (recording every 15 seconds from up to 8 satellites versus every 5 seconds from up to 12 satellites in the low and full power modes, respectively). Pilot work suggested that the high power mode was necessary to acquire data of a sufficient quality for the purposes of TPA tracking. The reduced battery life on high power mode meant that Trackstick batteries had to be replaced by the research assistant on a daily basis when participants attended their workplace.

To map its position internally, the Trackstick unit requires a stationary 'cold start' period of 15 minutes with an unobstructed view of the sky when turning the units on for the first time and after battery replacement. Standard GPS procedures also require a 'warm start' for accurate signal acquisition after extended signal loss has occurred (eg, entering and leaving a building), whereby the unit must remain stationary with an unobstructed view of the sky for 1 minute before continuing with usual activity. To reduce participant burden, the cold start initialization was completed by the research assistant before distribution to participants, and every time batteries were replaced. To ensure the units were functioning before delivery to participants, the research assistant also followed each cold start procedure with 15 minutes of movement with a clear view of the sky. Participants were requested to perform a warm start every time they left a building.

At the end of the measurement period, participants attended a second meeting with the research assistant to return the monitors and travel logs, and complete a questionnaire to elicit (1) participants' TPA patterns in the previous week using the International Physical Activity Questionnaire-Long Form (IPAQ-LF), ${ }^{18}$ (2) self-reported travel behaviors, and (3) general demographic information including residential address. Workplace address was defined as being at the North Shore campus of the Auckland University of Technology.

\section{Data Treatment}

GPS data were downloaded using TrackStick Manager 2.0.1 (Telespial Systems, Marina Del Rey, CA). Daily data files for each participant's journey to and from work were exported from TrackStick Manager 
to Google Earth (Google Inc., Mountain View, CA) to create spatial maps of overall travel for each GPS data file and to check for signal loss before further analyses. Participants' residential and workplace addresses were geocoded in Google Earth to assess agreement between reported start and finish locations and those in the GPS data file. In these cases, the commute route was included for further analyses if the distance between the mapped address and the GPS start- or end-point did not exceed $2.5 \mathrm{~m}$ from the property boundary (maximum distance of Trackstick imprecision). GPS data were excluded from further analyses if signal loss during the commute to/from place of work resulted in incomplete commute data as it was not possible to predict route taken during the period of signal drop out. ${ }^{19}$ Data were also excluded if the commute included travel outside the available GIS database region.

Using the Google Earth map as a reference, startand end-points of the commute route and the direction of commute were identified. Data pertaining to the commute route only were extracted into a Microsoft Excel (Microsoft Corp, Redmond, WA) file for analysis. Where possible, commutes both to and from participants' workplaces were included. To enable integration with accelerometer data, GPS commute route data were collapsed into 1-minute epochs by retaining the spatial location data from the first data point of each minute and deleting all other data points within that minute. For the final minute of the commute trip, the last data point of the minute was retained to ensure the spatial data for the destination was collected.

Accelerometer data were downloaded using Actical Version 2.04.0000 (Respironics Inc., Bend, OR). The Actical in-built batch processing function was used to extract csv files for each individual. These contained participant information, activity counts, energy expenditure, and activity intensity for each epoch. No predefined settings within the software were overwritten. To match the accelerometer data to the GPS data, the accelerometer epochs were collapsed to 1-minute intervals. The accelerometer data were then integrated into the GPS commute journey data file using Microsoft Access (Microsoft Corp, Redmond, WA). The participant ID, date and time fields in each file were used to match GPS and accelerometer epochs. Differences in descriptive information (ie, speed, duration, distance, and activity intensity gathered from the GPS and accelerometer data) for active and motorized commute trips (self-reported) were ascertained using oneway ANOVA with significance set at $\alpha=0.05$.

The resulting GPS/accelerometer commute journey data file was mapped in ArcView 9.2 (ESRI, Redlands, CA). ArcView is GIS software that enables the creation of a study-specific database containing layers (eg, census data, land utilization) of environmental features that may be associated with a behavior of interest, such as TPA. GIS software like ArcView allows for the querying, management, analysis, and visualization (eg, map-making) of spatial data, and thus is a valuable tool for studies investigating environmental associates of physical activity and TPA. While Google Earth is useful for querying and viewing spatial data, it does not provide the ability to analyze data, and compared with GIS software, it has limited visualization and mapping capabilities. Mapping data using ArcView provided spatial representations of physical activity accumulated at differing intensities (using Actical software-derived thresholds) for differing transport modalities (using questionnaire data with GPS-derived routes and speeds) throughout the commute routes. Home and work addresses were manually geo-coded using ArcView. Commute routes between participants' home and workplace addresses along the street network were plotted from the GPS data using the ArcView 9.2 Network Analyst Route function.

\section{Results}

Forty university employees enrolled in the study. Three participants did not complete the study protocol and were excluded from the study. Accelerometer, survey, and GPS data were collected from the remaining 37 participants, the characteristics of whom are provided in Table 1.

Table 1 Participant Characteristics

\begin{tabular}{lcc}
\hline Variable & N & (\%) \\
\hline Sex & & \\
$\quad$ Male & 10 & $(27)$ \\
Female & 24 & $(65)$ \\
$\quad$ No response & 3 & $(8)$ \\
Age group & & \\
20-30 years & 11 & $(29)$ \\
$31-40$ years & 7 & $(19)$ \\
$41-50$ years & 7 & $(19)$ \\
51-60 years & 6 & $(16)$ \\
No response & 6 & $(16)$ \\
\hline
\end{tabular}


Table 2 Descriptive Information for Quality of GPS Data Collected

\begin{tabular}{|c|c|c|}
\hline Variable & $\mathbf{N}$ & $(\%)$ \\
\hline Potential trips from GPS data & 259 & $(100)$ \\
\hline \multicolumn{3}{|l|}{ GPS trips lost due to } \\
\hline signal loss ${ }^{\mathrm{a}}$ & 26 & $(10)$ \\
\hline unit not worn & 4 & (1) \\
\hline no origin or destination in routes detected & 14 & $(5)$ \\
\hline noncollection of unit—weekday & 58 & $(22)$ \\
\hline noncollection of unit-weekend day & 68 & (26) \\
\hline problems downloading data & 2 & (1) \\
\hline device failure- unit dropped by participant & 3 & (1) \\
\hline lost device & 7 & (3) \\
\hline unexplained $^{\mathrm{b}}$ & 46 & $(18)$ \\
\hline commute route went outside the GIS database region & 2 & (1) \\
\hline \multicolumn{3}{|l|}{ GPS trips collected } \\
\hline from workplace to residence (evening) & 26 & $(10)$ \\
\hline from residence to workplace & 3 & (1) \\
\hline
\end{tabular}

Table 2 shows descriptive information for the GPS data collected and the number of journeys in each direction available for analysis. From a potential of 259 GPS commute trips from all participants, only 29 (11\%) trips were collected. Nearly half of the potential GPS-derived commute trips were lost due to an inability to contact participants or participants being unable to attend daily meetings for battery replacement. Other reasons for missing GPS data were: unexplained data loss (18\%), signal loss $(10 \%)$, no commute destination or origin in the GPS commute route (5\%), lost device (3\%), participant noncompliance $(1 \%)$, or hardware or software problems (2\%). Commonly, signal loss resulted in data points dispersed such that either the commute path was compromised and instead a 'crow fly' path was collected, data points were sporadic and did not allow a commute route to be determined, or no data points were shown in Google Earth. In 14 instances, the GPS route did not match reported journey destinations and origins. In total, 24 participants were excluded from further analysis due to erroneous, corrupt, insufficient, or missing data. For the remaining 13 participants, on average, 2 trips per participant were retained (range 1 to 5 trips), over an average of 2 days (range 1 to 4 days).

Self-reported travel modes from workplace (as identified from the travel logs) were collected for 153 of 259 possible days. Of those 153 days, in total 99 (65\%) journeys were motorized trips only ( $\mathrm{n}=97 \mathrm{car}, 2 \mathrm{bus}$ ), $34(22 \%)$ were TPA trips ( $\mathrm{n}=11$ walk, 23 cycle), and $20(13 \%)$ were a combination of motorized transport and TPA ( $\mathrm{n}=9 \mathrm{car} / \mathrm{walk}, 11 \mathrm{bus} / \mathrm{walk})$. Sixteen (16\%) of the motorized trips were captured via GPS during the journey from work ( $\mathrm{n}=15 \mathrm{car}, 1$ bus) and $9(26 \%)$ of the TPA journeys were acquired ( $\mathrm{n}=2$ walk, 7 bike). For trips to work, 152 self-reported travel modes were collected, with $100(66 \%)$ being motorized trips only $(\mathrm{n}=97 \mathrm{car}, 2 \mathrm{bus,}$ 1 bus/car), 32 (21\%) TPA only ( $\mathrm{n}=11$ walk, 21 cycle), and $20(13 \%)$ a combination of motorized and TPA trips TPA ( $\mathrm{n}=9 \mathrm{car} /$ walk, 10 bus/walk, $1 \mathrm{car} / \mathrm{bus}$ ).

Average overall trip duration assessed by GPS was 25.5 minutes. Although limited GPS data were collected, clear differences in reported TPA and motorized transport modes were observed, with shorter distances, longer trip durations, and slower speeds for TPA than for motorized trips (Table 3). Travel speed was the only variable for which a significant difference was found between motorized and active transport modes. It is likely that the differences in trip duration and distance would be statistically significant with a greater number of observations.

As the availability of GPS data determined the corresponding accelerometer information to be imported into the GIS database, the amount of matched GPSaccelerometer data were substantially lower than the total amount of accelerometer data. For the accelerometer data that were retained, the proportion of commute trip duration spent in sedentary, light, and moderate-to-vigorous physical activity for participants was $74 \%, 20 \%$, and $6 \%$, respectively; these figures differed only slightly by travel mode and differences were not statistically significant.

GIS maps of commute routes traveled using the GPS data and corresponding physical activity intensities from the accelerometer data were successfully generated 
Table 3 Physical Activity Intensity and Travel Differences by TPA and Motorized Transport Modes

\begin{tabular}{|c|c|c|c|c|c|c|}
\hline & \multicolumn{3}{|c|}{ GPS-derived commute mode descriptive information } & \multicolumn{3}{|c|}{$\begin{array}{l}\text { Accelerometer-derived physical activity } \\
\text { intensity }\end{array}$} \\
\hline & $\begin{array}{c}\text { Average (SD) } \\
\text { trip duration } \\
\text { (minutes) }\end{array}$ & $\begin{array}{c}\text { Average }(\mathrm{SD}) \text { trip } \\
\text { distance }(\mathrm{km})\end{array}$ & $\begin{array}{l}\text { Average (SD) } \\
\text { speed (mph) }\end{array}$ & SED (\%) & LPA (\%) & MVPA (\%) \\
\hline TPA & $35.5(21.2)$ & $5.4(3.0)$ & $8.4(4.0)$ & 75 & 16 & 9 \\
\hline Motorized & $21.0(34.0)$ & $7.9(3.3)$ & $20.6(9.3)^{*}$ & 73 & 22 & 5 \\
\hline
\end{tabular}

Abbreviations: GPS, global positioning system; LPA, light physical activity; MVPA, moderate-to-vigorous physical activity; SD, standard deviation; SED, sedentary; TPA, transport-related physical activity.

$* P<.01$.

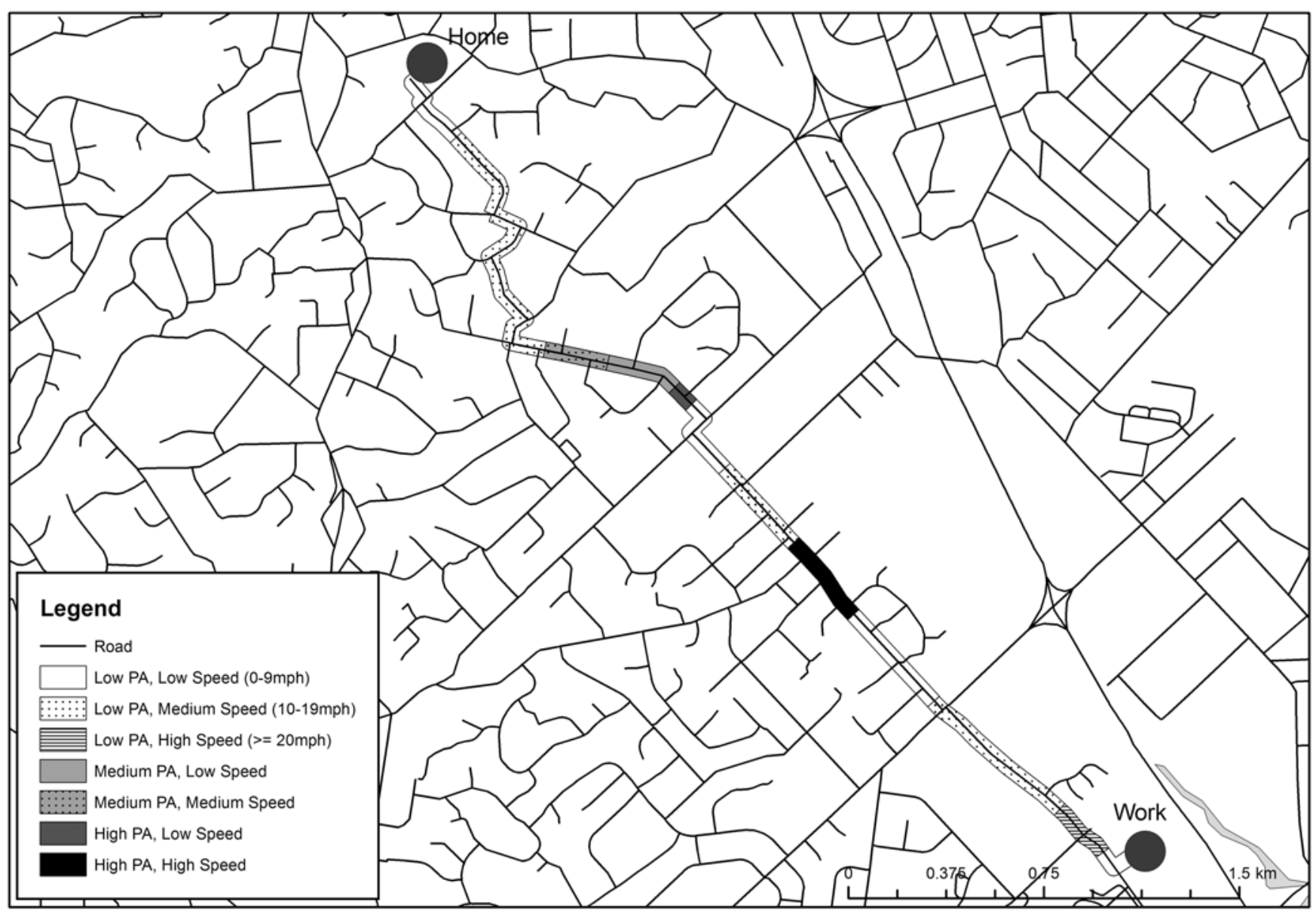

Figure 1 - Example of one participant's commute route from home to workplace and corresponding physical activity intensity and speeds.

for all participants. Figure 1 shows an example of one participant's commute from work to residence using the integrated GPS and accelerometer data. Self-reported mode of travel was bicycle. The route generated shows GPS-determined speeds throughout the route, as well as accelerometer-deemed physical activity intensity during the commute. As can be seen from Figure 1, the self-reported mode of travel can be confirmed by using the objective measures; the traveling speeds measured using the GPS denote travel by cycling, while the physical activity intensity is indicative of the participant being mostly active throughout the commute. Differing physical activity intensities during the commute are indicative of cycling for transport, whereby there are periods of low activity (as would be expected when sitting on a bike seat and cycling at a low intensity), and medium to high inten- 
sity (likely due to vigorous cycling or cycling out of the saddle). Actual cycling behaviors cannot be definitively determined using GPS or accelerometry; however, the combined consideration of GPS and accelerometer data enables researchers to hypothesize these behaviors with a greater degree of certainty than using only 1 approach.

\section{Discussion}

GPS technology is now widely available for assessing spatial location of physical activity, yet to our knowledge no research has combined GPS with other objective measures of physical activity to examine traveling modalities for work commutes. While our limited GPS data inhibited detailed exploration of these relationships, differences between motorized and active modes of transport were observed and occurred in the expected directions. In addition, the relatively short distances found for motorized trips have identified the opportunity for intervention and promoting TPA for short commute trips. Interestingly, no difference in physical activity intensity by travel mode was found. This finding is likely a result of cycling being the most prevalent mode for TPA trips captured $(78 \%)$, and an inability of hip-mounted accelerometers to adequately capture activity intensity of cycling due to limited movement occurring at the trunk region.

The purpose of this paper is to be illustrative rather than definitive, and in doing so provide an overview of the issues of relevance to researchers considering using GPS units to assess physical activity patterns. We identified a number of issues related to the practicalities of collecting physical activity and travel data using accelerometers and GPS, and the integration of these data with a GIS database. We also found that the Trackstick GPS devices had to be set to a high-power mode to improve data quality and accuracy, thereby requiring daily battery replacement. This process resulted in considerable participant and researcher burden, and ultimately substantial data loss. Considering these constraints, the Trackstick units appear to be best suited for tracking physical activity patterns over shorter measurement durations ( $<24$ hours). Irrespective of data collection time frame, signal loss can compromise the quality of data collected. In choosing the most appropriate GPS unit for research purposes, this potential data loss is an important consideration, in conjunction with overall unit accuracy, warm and cold start requirements, unit cost and size, and data collection capacities.

A summary of the methodological issues and recommendations related to the use of GPS units with GIS and accelerometer data are presented below.

- This research focused on TPA trips to/from participants' place of employment to/from their residence. This was relatively straightforward to determine with our small sample size and travel log procedures where self-reported waking times could be extrapolated to the GPS data. Larger studies investigating multiple trips throughout the day would need to develop data treatment rules and algorithms such as Stopher et a ${ }^{19}$ to enable the categorization of trip purpose for large datasets. Travel diaries may provide the level of detail required, however these are relatively burdensome for the participant and data collected may be subjective or biased.

- Data downloaded from TrackStick units does not include information pertaining to the time zone setup (eg, Greenwich Mean Time [GMT], GMT+12 hours, etc.). If researchers are interested in integrating GPS data with other measures using time as the common denominator, it is highly recommended to standardize and record the time zone setup used across different units.

- Stationary time periods before commencing journeys will be useful in limiting the volume of data lost due to poor matches between reported and GPS determined start locations. Performance parameters of the GPS chipset used in the unit will likely determine the amount of time needed for accurate satellite acquisition. Regardless, participants should ideally be encouraged to remain stationary until the unit has acquired signals from at least 4 satellites before commencing their journey. Whether participant compliance can be achieved with such protocols remains to be determined. It is possible that the information provision regarding the importance of adequate satellite acquisition for data collection may be sufficient; however, participant incentives may also be required for improved likelihood of compliance.

- The potential for data loss due to problems with signal acquisition must be considered when identifying appropriate activity measurement tools. Furthermore, there is potential that signal loss may be greater in other settings with tall buildings, large land marks, or dense foliage present, as these features have been shown to block satellite contact with GPS units. ${ }^{19}$ If the TrackStick units are to be used, the sample size should be increased accordingly to ensure sufficient data are collected.

- The integration of GPS into GIS databases is restricted to locations for which GIS database information is available, and considerable data loss may occur where participants leave the boundaries of the GIS database. Accordingly, researchers need to either ensure the study sample will remain within the area for which GIS data exists, or ensure sufficient geographic spread of existing GIS databases.

- The inability of hip worn accelerometers to accurately detect cycling activities is a well known limitation of these technologies ${ }^{20}$ and continued use when assessing TPA journeys that contain a high proportion of cycling journeys may misrepresent the actual intensity of TPA. Therefore other devices with similar or better levels of reliability and validity capable of objectively assessing physical activities including cycling activities should be explored in future studies. 
Advancing technologies will likely result in improvements in GPS equipment in the near future, and this will have positive implications on prospective research. Emerging technologies such as radiofrequency identification tags (RFID) have been suggested as one potential way to overcome the shortcomings of GPS in built up urban areas. ${ }^{21}$ These units have their own limitations as well: the researcher is required to set up the receiver stations throughout the urban environment, and to date these units have been relatively untested in their capacity to be integrated into public health research.

An encouraging development is the iPhone $3 \mathrm{G}$ (Apple, Cupertino, CA) which improves data acquisition by using Wi-Fi hot spots and cell phone receiver towers to triangulate position when a GPS-satellite fix is not possible, with little effect on battery life. This technology allows for real-time continuous spatial tracking, irrespective of GPS satellite acquisition, but is limited by the presence of Wi-Fi hot spots and cell phone receiver towers. Increasing availability and affordability of new, improved technologies will enable researchers to understand the complexities of physical activity behaviors in a detailed and objective manner, as well as identify determinants and health outcomes of differing physical activity dimensions and profiles.

\section{Conclusion}

While this feasibility study highlights the complications that can arise when using GPS to monitor free-living behaviors, we suggest that the potential strengths of GPS monitoring to gather objective measures of human motion and TPA warrant further refinement of GPS as a research instrument. Integration of GPS, accelerometer, and GIS provides a depth of knowledge superior to current self-report approaches to understanding travel and physical activity behaviors and their relationship with the built environment.

\section{Acknowledgments}

This study was funded by a Building Research Capacity in the Social Sciences Grant (Grant Number: PR57855) and National Heart Foundation Small Project Grant (Grant Number: 1299). MO and HMB are supported by National Heart Foundation of New Zealand Research Fellowships.

\section{References}

1. Badland H, Schofield G. The built environment and transport-related physical activity: what we do and do not know. J Phys Act Health. 2005;2(4):433-442.

2. Transportation Research Board and Institute of Medicine of the National Academies. Does the Built Environment Influence Physical Activity? Examining the Evidence. Washington, DC: Transportation Research Board, Institute of Medicine of the National Academies; 2005.

3. Sullivan C, Oakden J, Young J, Butcher H, Lawson R. Obstacles to Action: A Study of New Zealanders'Physical Activity and Nutrition. Wellington, New Zealand: Sport and Recreation New Zealand; 2003.
4. Hayashi T, Tsumura K, Suematsu C, Okada K, Fuji S, Endo G. Walking to work and the risk for hypertension in men: The Osaka Health Survey. Ann Intern Med. 1999;130:21-26.

5. Hu G, Pekkarinen H, Hanninen O, Yu Z, Guo Z, Tian H. Commuting, leisure-time physical activity, and cardiovascular risk factors in China. Med Sci Sports Exerc. 2002;34(2):234-238.

6. Hu G, Pekkarinen H, Hanninen O, Tian H, Guo Z. Relation between commuting, leisure time physical activity and serum lipids in a Chinese urban population. Ann Hum Biol. 2001;28(4):412-421.

7. Hamer M, Chida Y. Active commuting and cardiovascular risk: a meta-analytic review. Prev Med. 2008;46(1):9-13.

8. Pikora TJ, Giles-Corti B, Kkuiman MW, Bull FC, Jamrozik K, Donovan RJ. Neighborhood environmental factors correlated with walking near home: using SPACES. Med Sci Sports Exerc. 2006;38(4):708-718.

9. Badland H, Schofield G, Garrett N. Travel behavior and objectively measured urban design variables: associations for adults traveling to work. Health Place. 2008;14(1):8595.

10. Duncan M, Mummery W. GIS or GPS? A comparison of the two methods for assessing route taken during active transport. Am J Prev Med. 2007;33(1):51-53.

11. Sallis JF, Saelens BE. Assessment of physical activity by self-report: status, limitations, and future directions. Res Q Exerc Sport. 2000;71(2):1-14.

12. Rodriguez D, Brown A, Troped P. Portable global positioning units to complement accelerometry-based physical activity monitors. Med Sci Sports Exerc. 2005;37(11, Suppl):S572-S581.

13. Mackett R, Brown B, Gong Y, Kitazawa K, Paskins J. Children's independent movement in the local environment. Built Environ. 2007;33(4):454-468.

14. Esliger DW, Tremblay MS. Technical reliability assessment of three accelerometer models in a mechanical setup. Med Sci Sports Exerc. 2006;38(12):2171-2183.

15. Heil DP, Klippel NJ. Validation of energy expenditure prediction algorithms in adults using the Actical electronic activity monitor. Med Sci Sports Exerc. 2003;35(5):S284.

16. Heil DP. Predicting activity energy expenditure using the Actical activity monitor. Res Q Exerc Sport. 2006;77(1):6480.

17. Matthews CE, Ainsworth BE, Thompson RW, Bassett DR, Jr. Sources of variance in daily physical activity levels as measured by an accelerometer. Med Sci Sports Exerc. 2002;34(8):1376-1381.

18. Craig CL, Marshall AJ, Sjöström M, et al. International Physical Activity Questionnaire: 12-country reliability and validity. Med Sci Sports Exerc. 2003;35(8):1381-1395.

19. Stopher P, FitzGerald C, Zhang J. Search for a global positioning system device to measure person travel. Transp Res. In press.

20. Welk GJ. Use of accelerometry-based activity monitors to assess physical activity. In: Welk G, ed. Physical Activity Assessments for Health-Related Research. Champaign, IL: Human Kinetics; 2002.

21. Freedson PS, Brendley K, Ainsworth BE, Kohl HW, Leslie E, Owen N. New techniques and issues in assessing walking behavior and its contexts. Med Sci Sports Exerc. 2008;40(7, Suppl):S574-S583. 